\title{
Prevention of Virus Propagation in Mobile Network
}

\author{
Balasundaram.R \\ MTECH Student, \\ Department of Computer \\ Science \& Engineering, Dr.SJS \\ Pauls Memorial College of \\ Engineering and Technology, \\ Pondicherry University, \\ Pondicherry, India
}

\author{
Ezhumalai.P \\ Assistant Professor, \\ Department of Computer \\ Science \& Engineering, Dr.SJS \\ Pauls Memorial College of \\ Engineering and Technology, \\ Pondicherry University, \\ Pondicherry, India
}

\author{
Balamurugan.G \\ MTECH Student, \\ Department of Computer \\ Science \& Engineering, \\ Manakula Vinayagar Institute \\ of Technology, \\ Pondicherry University, \\ Pondicherry, India
}

\begin{abstract}
Infections and malwares have the capacity to spread beginning workstation systems into versatile systems with the fast improvement of brilliant phone clients .Currently, there are three disease vectors utilized by portable malware to spoil versatile. A Bluetooth affiliation permits document exchange if the telephone's Bluetooth stack helps OBEX trade - which most telephones do. MMS messages can encase not just sound and pictures, they can additionally convey executables. Some cell telephone infections utilize this disease vector excessively - e.g., the infections from the Commwarrior precursors. It's simpler to reject a MMS message than it is to decline a Bluetooth record sending, in light of the fact that the interest comes earlier. In any case, the message by and large originates from some individual you know, as the infection sends itself to the cell telephone on the tainted telephone's phonebook - in this way, you're more prone to open it. Finally, in the event that you embed a contaminated MMC card into your telephone, different projects on it can mechanically execute. This defilement vector is likewise utilized by the Commwarrior infection family. These are the contamination vectors as of now being used. The correspondence channel that could be utilized to exchange executable substance might be utilized to send an infection. These comprise of Wi-Fi interchanges, skimming the Web, synchronizing with a contaminated PC etc. We propose a two-layer system model for mimicking infection spread through in participation of Bluetooth and SMS. We examine two methods for controlling portable infection engendering. The primary method is pre immunization and versatile scattering systems.
\end{abstract}

\section{Keywords}

Bluetooth, I-Fi, SMS, MMS, PC, MMC, BT-Based

\section{INTRODUCTION}

Infection can even stick remote administrations through dissemination a huge number of spam messages, and reduction the nature of voice correspondence.

The substantial spread can arranged into two ways

1) Gauges the scale of an infection episode before it happens actually and

2) Assess as good as ever counter measures for limiting infection proliferation. We propose a two-layer system model for portraying BT-based and SMS-based infections, which flow through Bluetooth and Short/Multimedia Message Services.

There are two sorts of human conduct. The First sort is operational conduct and the second sort is versatile conduct (portability), are considered in our individual-based model.
The impacts of system structure on infection proliferation planned to how human practices influence the spread motion of versatile infections. The recognition engineering is utilized to identification reason. Discovery advances can perform ensure tainted telephones from sending contaminated messages focused around framework calls arrangements and Apis .They won't have the capacity to catch new infections because of the limit of antivirus learning. The Service suppliers need to scatter notices or patches to advanced mobile phones. The propose another system that can productively send patches to the same number of versatile hub as likely, even in vast scale progressively developing systems. We plan a versatile scattering method by augmenting nearby receptive practices of substances. The objective of our work is triple:

1) To reveal some key components in deciding versatile infection spread through our two-layer system proliferation model.

2) To investigate the impacts of operational examples and portability designs on versatile infection spread

3) To analyze two techniques for limiting infection spread in versatile systems, i.e., pre immunization and versatile patch dispersal procedures drawing on the approach of selfsufficiency situated processing (AOC).

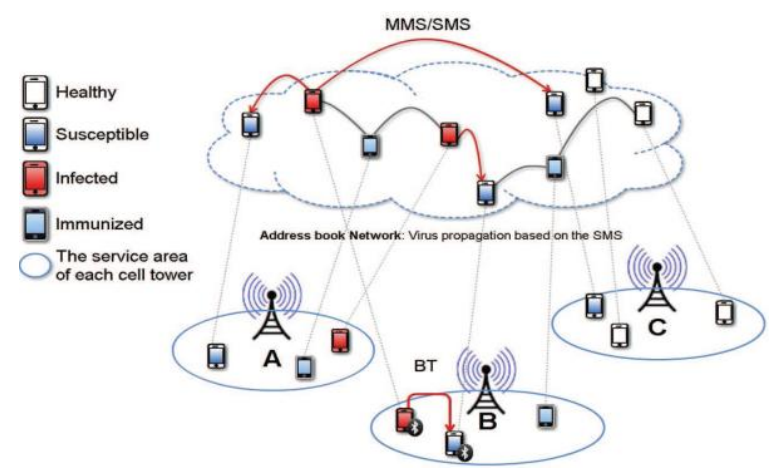

Fig. 1: A two-layer network model for simulating mobile virus propagation

\section{IMPLEMENTATION AOC SPECIFICATION}

In the current framework absence of system layer model to actualize AOC determination. The new infection can't be recognized by the antivirus or other security application. A BT-based infection is a neighborhood contact driven infection since it contaminates different telephones just through Bluetooth and Wi-Fi gadgets inside a short radio extent. In 
light of the constrained transmission scope of a Bluetooth gadget, human versatility assumes a critical part in BT-based infection spread. Hard to recognize the bundle sending or hubs development over the system. Arranging the hub and sending procedure are difficult to actualize. In the event that the characterization doesn't perform splendidly then the second and last process can't actualize. The controlling methodology performs the blocking procedure. While we don't execute the limiting methodology it permits the infection and malware over the system.

\subsection{Self-rule Oriented Computing:}

Our proposed two-layer system model, we have inspected two procedures for controlling SMS based Virus engendering that is focused around the strategy of self-sufficiency turned figuring. The Autonomy Oriented Computing - based pre immunization system is fit for limiting versatile infection engendering by ensuring some exceedingly associated telephones, while the AOC-based dispersal technique can advance security notices or patches to however many telephones as could reasonably be expected with a low correspondence cost so as to help them recuperate or maintain a strategic distance from the potential harms of portable infections. The pre immunization procedure predicts the cell telephone infection and malware to maintain a strategic distance from the danger. The patch dispersal systems proficiently send the security notices.

We reenact one kind of operational conduct, i.e., whether a client opens a suspicious message. The likelihood of clicking on a suspicious connection could be utilized to reflect and evaluate the security consciousness of a client. Similar to conduct has been utilized to recreate email infection engendering.

\subsection{ADVANTAGE}

- The Communication Cost is lower.

- The Higher Coverage Rate (maximum distribution)

\section{TECHINQUES FOR PREVENTION IN MOBILE}

\subsection{VIRUS PROPAGATION}

The infection is an area of programming code that runs or obstructs our running application without any power. Malware is a mantle term that is utilized to outline various pernicious sorts of programming, including adware, spyware, infections, Trojans and that's only the tip of the iceberg.

Spyware is a particular kind of malware, yet is very not the same as most different pernicious programming due to what it is intended to do. The infection Propagation could be characterized into two sort's in particular BT-based infection spread and the SMS based infection proliferation. Spyware's motivation originates from its name, and is intended to watch your activities. Spyware will take a gander at what destinations you visit, and in more amazing cases, track what you write to take your passwords and individual data.

\subsection{HUMAN MOBILITY}

We have utilized a homogenous model to reproduce BT-based infection proliferation in each one tower; clients' diverse voyaging examples will result in distinctive element spreading techniques.
Three fundamental aspects for example influencing BT-based infection engendering:

' whether or not a client moves at a given hour

'users' come back to went by spots at whenever

- traveling separations of a client

\subsection{BT-BASED PROPAGATION PROCESS}

Infections that spread via email or SMS message could be examined moderately effortlessly by a system administrator utilizing existing innovations (i.e. by contrasting them with a database of known malware).the BT - Based infection spread gauges the separation between one hub to an alternate hub. The engendering of one hub to an alternate hub is called as voyaging separation.The voyaging separations of a client are created by a force work, a toll flight procedure and an arbitrary walk.

The short extend infections that spread from telephone to telephone by means of Bluetooth and Wi-Fi is more slippery. Cell telephones don't fundamentally have the registering force to output all approaching message.

\subsection{SMS BASED PROPAGATION PROCESS:}

On the off chance that a telephone is tainted by a SMS-based infection, the infection naturally sends its duplicates to different telephones focused around the location book of the contaminated telephone. At the point when clients get a suspicious message from others, they may open or erase it focused around their security mindfulness and information about the dangers of versatile infections.

The characterization of SMS Based infection Propagation,

- if a client opens a tainted message; the telephone of this client is contaminated and naturally sends infections to all telephones focused around its address book

- if a client does not open a tainted message, it is accepted that the client with higher security mindfulness erases this contaminated message

- An tainted telephone conveys infections to different telephones just once, after which the contaminated telephone won't convey infections.

\subsection{PRE-IMMUNIZATION STRATEGY}

While most malware could be filtered for and uprooted by an antivirus program, spyware is a bit better at concealing itself. To evacuate spyware, an upgraded antispyware program generally does the trap; antispyware projects are not the same as antivirus projects, so it's useful to have one of each on your machine. Antispyware programs likewise by and large don't screen your machine, and may just get spyware amid framework filters. A pre immunization procedure to secure systems before infection spread. 


\subsection{PATCH DISSEMINATION STRATEGY}

A patch document is a change made by the producer to purpose programming issues and brings about a significant improvement. A break document is an illicit change that permits an application to be run that has not been acquired by the client. We propose a versatile dispersal system focused around the procedure of independence turned figuring (AOC) with a specific end goal to proficiently send security notices or patches to the majority of telephones with a generally lower correspondence cost.

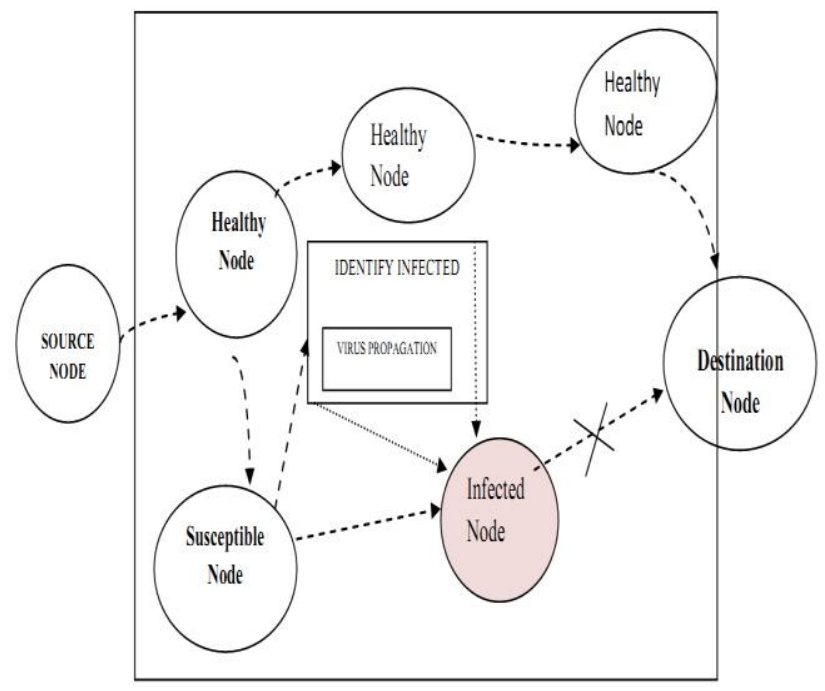

Fig. 2: System Archietecture of Mobile Virus Propagation

\section{MODULES}

$\begin{array}{ll}\checkmark & \text { NODES FLOW } \\ \checkmark & \text { VIRUS PROPAGATION } \\ \checkmark & \text { RESTRAINING PROCESS }\end{array}$

\subsection{NODES FLOW:}

A hub is any gadget associated with a workstation system. Hubs might be workstations, particular computerized colleagues (Pdas), mobile phones, or different other system apparatuses. On an IP system, a hub is any gadget with an IP address. In this Network demonstrates the all hubs to group the solid telephone, suspected telephone, tainted telephone and immuned telephone. By this recognizable proof we can go before the further process.

\subsection{VIRUS PROPAGATION}

The Nodes are put in the system and move from source to objective, In this Virus Propagation ordering the sound telephone, powerless telephone, and contaminated telephone. By this arrangement we can dodge the future hazard, for example, BT-infection engendering and SMS infection spread.

\subsection{RESTRAIINING PROCESS}

The Restraining is a methodology of obtaining to hold or piece the undesirable administration. Here, the undesirable administration characterizes the infection and malware bundles as a hub that engenders through system. Utilizing the infection spread condition to piece the BT -based infection engendering and the SMS based infection proliferation

\section{CONCLUSION}

We have actualized two layer system models for reproducing and dissecting the engendering flow of SMS-based and BTbased infections. In this paper human conduct might be isolated into two models. The two models are operational conduct and the versatile conduct. Utilizing these two models we can distinguish the revealed infection over system. The conduct is key part to send security warnings. The hub stream, recognizing infection engendering and controlling methodology demonstrates the usage part.

\section{REFERENCES}

[1] D.-H. Shi, B. Lin, H.-S. Chiang and M.-H. Shih, "Security aspects of mobile phone virus: A critical survey," Industrial Management and Data System, vol. 108, no. 4, pp. 478-494, 2008.

[2] H. Kim, J. Smith, and K. G. Shin, "Detecting energygreedy anomalies and mobile malware variants," Proceedings of the $6^{\text {th }}$ International Conference on Mobile Systems, Applications, and Services (MobiSys08), pp. 239-252, 2008.

[3] L. Xie, H. Song, T. Jaeger, and S. Zhu, "A systematic approach for cell-phone worm containment," Proceedings of the 17th International World Wide Web Conference (WWW08), pp. 1083-1084, 2008.

[4] N. Xu, F. Zhang, Y. Luo, W. Jia, D. Xuan, and J. Teng, "Stealthy video capture: A new video-based spyware in 3G smart phones," Proceedings of the 2nd ACM Conference on Wireless Network Security (WiSec09), pp. 69-78, 2009.

[5] G. Yan and S. Eidenbenz, "Modeling propagation dynamics of Bluetooth worms (extended version)," IEEE Transactions on Mobile Computing, vol. 8, no. 3, pp. 353-367, 2009.

[6] C. Gao, J. Liu, and N. Zhong, "Network immunization and virus propagation in email networks: experimental evaluation and analysis," Knowledge and Information Systems, vol. 27, no. 2, pp. 253-279, 2011.

[7] P. Wang, M. C. Gonzalez, C. A. Hidalgo, and A.-L. Barabasi,"Understanding the spreading patterns of mobile phone viruses, "Science, vol. 324, no. 5930, pp. 1071-1076, 2009.

[8] S. Cheng,W. C. Ao, P. Chen, and K. Chen, "On modeling malware propagation in generalized social networks," IEEE Communications Letters, vol. 15, no. 1, pp. 25-27, 2011.

[9] P. De, Y. Liu, and S. K. Das, "An epidemic theoretic framework for vulnerability analysis of broadcast protocols in wireless sensor networks," IEEE Transactions on Mobile Computing, vol.8,no.3,pp. 413425, 2009.

[10] P. De, Y. Liu, and S. K. Das, "Deployment aware modeling of node compromise spread in wireless sensor networks using epidemic theory," ACM Transactions on Sensor Networks,vol.5,no.3,pp.1-33, 2009.

[11] M. C. Gonzalez, C. A. Hidalgo, and A. L. Barabasi, "Understanding individual human mobility patterns," Nature, vol. 453, no.7196, pp. 779-782, 2008.

[12] C. C. Zou, D. Towsley, and W. Gong, "Modeling and simulation study of the propagation and defense of 
internet e-mail worms, "IEEE Transaction on Dependable and Secure Computing, vol.4, no.2, pp. 105-118, 2007.

[13] L. Xie, X. Zhang, A. Chaugule, T. Jaeger, and S. Zhu, "Designing system-level defenses against cellphone malware," Proceedings of the 28th IEEE International Symposium on Reliable Distributed Systems (SRDS09), pp. 83-90, 2009.

[14] A. Bose, X. Hu, K. G. Shin, and T. Park, "Behavioral detection of malware on mobile handsets," Proceedings of the 6th International Conference on Mobile Systems, Applications, and Services (MobiSys08),pp. 225-238, 2008 .
[15] F. Li, Y. Yang, and J. Wu, "CPMC: An efficient proximity malware coping scheme in Smartphone-based mobile networks, "Proceedings of the 29th IEEE International Conference on Computer Communication (INFOCOM10), pp. 2811-2819, 2010.

[16]G.Zyba,G.M.Voelker,M.Liljenstam,A.Mehes,andP.Johans son, "Defending mobile phones from proximity malware, "Proceedings of the 28th IEEE International Conference on Computer Communication (INFOCOM09), pp. 1503$1511,2009$. 